\title{
LAS CUENTAS SATÉLITES DEL TURISMO EN EL PERÚ, AÑO 2013: MUSEOS, RESTOS ARQUEOLÓGICOS Y SITIOS NATURALES DE LAS REGIONES DE AREQUIPA, CUSCO, LAMBAYEQUE Y LA LIBERTAD
}

\author{
PERU TOURISM SATELLITE ACCOUNTS, YEAR 2013: \\ MUSEUMS, ARCHAEOLOGICAL RESTS AND \\ NATURAL SITES OF THE REGIONS OF AREQUIPA, \\ CUSCO, LAMBAYEQUE AND LA LIBERTAD
}

Marcia Adriana Iberico Díaz Investigadora del Observatorio Turístico del Perú, Universidad de San Martín de Porres, Perú (Región Lambayeque y La Libertad)

Alejandro Málaga Nuñez-Zeballos Investigador del Observatorio Turístico del Perú, Universidad de San Martín de Porres, Perú (Región Arequipa)

José Manuel Enrique Marsano Delgado Director del Observatorio Turístico del Perú, Universidad de San Martín de Porres, Perú

María Olivia Pachas Fuentes Investigadora del Observatorio Turístico del Perú, Universidad de San Martín de Porres, Perú

José Pazos Miranda

Investigador del Observatorio Turístico del Perú, Universidad de San Martín de Porres,

Perú (Región Cusco) 


\title{
RESUMEN
}

Se describe el trabajo realizado por el Observatorio Turístico del Perú de la Escuela Profesional de Turismo y Hotelería de la Universidad de San Martín de Porres, que plantea como objetivo determinar la riqueza generada en el derrotero económico de los museos, restos arqueológicos y sitios naturales de las regiones de Arequipa, Cusco, Lambayeque y La Libertad. Además, se presenta antecedentes referentes a las investigaciones realizadas, así como deficiencias tanto desde el punto de vista cuantitativo como cualitativo en el sector turístico.

Para efectos de la medición del PIB de cada una de las unidades de producción correspondiente se utilizó el método de la retribución a los factores de producción.

Palabras clave: producto interno bruto, valor bruto de la producción, retribución a los factores de producción, trabajadores formales.

\begin{abstract}
They describes the work carried out by the Observatory Tourist of Peru of the Professional School of Tourism and Hospitality of the University of San Martin de Porres, they posed as an aim to determine the wealth generated in the economic course of the museums, archaeological rests and natural sites of the regions of Arequipa, Cusco, Lambayeque and La Libertad. In addition, it arises from background relating to the investigations carried out, as well as, differences from the quantitative and qualitative point of view in the tourism sector.
\end{abstract}

For the purposes of the measurement of the GDP of each of the corresponding production, units used the method of remuneration to the factors of production.

Keywords: gross domestic product, the gross value of production, compensation to the factors of production, formal workers.

\section{Introducción}

El Observatorio Turístico del Perú es una entidad de la Universidad de San Martín de Porres que viene realizando estudios económicos tendientes a conocer las cuentas satélites de los principales lugares turísticos del Perú. Es por ello que se plantea, como objetivo de esta investigación, determinar la riqueza generada en el derrotero económico de los museos, restos arqueológicos y sitios naturales en las regiones de Arequipa, Cusco, Lambayeque y La Libertad, independientemente al Producto Interno Bruto (PIB) generado. Asimismo, se establece el grado de ocupabilidad, empleabilidad e información de utilidad para la toma de decisiones. 


\section{Objetivos}

- Determinar la riqueza generada en el derrotero económico de las museos, restos arqueológicos y sitios naturales, ubicados en los destinos turísticos de Arequipa, Cusco, Lambayeque y La Libertad.

- Acopiar información científica necesaria para profundizar el estudio de las cuentas satélites del sector en las regiones turísticas del Perú.

- Proporcionar los indicadores necesarios para el sector turismo.

\section{Antecedentes}

Las investigaciones realizadas en el sector turístico, se han enfocado en la demanda, especialmente en los mercados emisores (perfiles) y es inexistente el análisis de la riqueza económica generada entre la demanda y la oferta turística, la misma que, en este caso, está referida a los museos, restos arqueológicos y sitios naturales. Como consecuencia de ello, el Observatorio Turístico del Perú notó las siguientes deficiencias tanto desde el punto de vista cuantitativo como cualitativo en el sector:

- Primera deficiencia. La estandarización de las cifras originadas por la demanda y la oferta turística. Ello lleva a la estimación de promedios, más no a cálculos sistematizados que nos permita saber a ciencia cierta las verdaderas cifras.

- Segunda deficiencia. Carencia de investigaciones referidas al nivel de riqueza generada y la distribución de la misma (PIB) por estos recursos y su capacidad de generación de trabajo.

- Tercera deficiencia. La carencia de elementos sumamente finos para cubrir las necesidades de información adecuadas para las autoridades estatales del sector y la de los empresarios privados para contar con una adecuada información en la toma de decisiones de futuras inversiones en el sector turismo.

Presentaremos brevemente dos casos en los que se ha implementado las cuentas satélites y que apuntan a metodologías similares a las que proponemos en el presente documento:

Primer caso:

Chile. El Observatorio de Turismo Regional de Antofagasta, como una forma de contribuir a la caracterización del sector turismo, ha desarrollado la primera Cuenta Satélite de Turismo Regional de Antofagasta (CSTRA) con el fin de aportar datos sobre el impacto que la industria del turismo tiene sobre la economía.

En 1999 Chile presentó la primera Cuenta Satélite de Turismo (CST) con datos del año 1996, y en el 2007 se presentaron los resultados para el año 2003, la proyección para el 2004 y la estimación para el 2005, mostrando así, que las autoridades a nivel nacional consideran importante el desarrollo de la CST, como herramienta de caracterización y medición del impacto de la actividad sobre la generación de 
producción y su vinculación con otros sectores de la economía, empleo e ingresos. Hoy en día, la necesidad de entender la dinámica del turismo en la economía regional, aportando mejor información para apoyar al proceso de decisión a todo nivel, es un punto crucial cuando los recursos son escasos y las necesidades de generar un desarrollo sustentable son una prioridad. Por esto el OTRA ha comenzado a desarrollar, en base a estadística básica de fuentes secundarias, la primera Cuenta Satélite de Turismo para la Región de Antofagasta (CSTRA) ${ }^{1}$.

Segundo caso:

España. La Cuenta Satélite del Turismo de España (CSTE) está compuesta por un conjunto de cuentas y tablas, basadas en los principios metodológicos de la contabilidad nacional, que presenta los distintos parámetros económicos del turismo en España, para una fecha de referencia dada. Comprende tres tipos de elementos:

1. Cuentas y tablas de oferta, en las que se trata de caracterizar la estructura de producción y costes de las empresas turísticas.

2. Tablas de demanda, en las que se trata de caracterizar, desde el punto de vista económico, los diferentes tipos de turistas, el turismo nacional frente al internacional, el tipo de bienes y servicios demandados, etc.

3. Tablas que interrelacionan la oferta con la demanda, que permiten obtener unas mediciones integradas de la aportación del turismo a la economía a través de variables macro como el PIB, la producción o el empleo.

Cabe notar que la Organización Mundial de Turismo (OMT), la Comisión de Comunidades Europeas (EUROSTAT), la Organización de Cooperación y Desarrollo Económico (OECD) y la División Mundial de Estadística de las Naciones Unidas, han venido trabajando desde la década de 1970 en el desarrollo de un sistema de medición que permita caracterizar el turismo y hacer comparable esta actividad a nivel mundial, dada la carencia de la contabilización de esta actividad en el Sistema Nacional de Cuentas.

\section{Marco Teórico}

Marsano (2003), sostiene que el Sistema de Cuentas Nacionales permite elaborar las cuentas que miden la actividad económica en su conjunto a través de la medición del Producto Interno Bruto (PIB). Pero como es obvio, no solo se limita a medir este agregado sino que también mide un amplio conjunto de variables que explican la actividad de la producción, el ingreso generado por esta actividad, la distribución de ese ingreso entre los diferentes propietarios de los factores productivos, los gastos de los consumidores intermedios y finales, la producción que se exporta y la que se requiere importar, entre otras importantes variables. Y, una vez determinado el ingreso, cuánto es lo que después de consumir queda disponible para el ahorro, y en qué instrumentos financieros queda invertido.

\footnotetext{
${ }^{1}$ http://sites.google.com/site/otraucn/investigacion/cstr
} 
Para efectuar estas mediciones las normas internacionales nos ofrecen una forma de organizar la información pudiendo establecer unidades de análisis como los establecimientos e industrias, por un lado, y sectores por otro.

Lo antes mencionado es lo que se denomina el marco central de las cuentas nacionales. Hasta aquí, este esquema en general no modifica el Sistema de las Cuentas Nacionales; sin embargo, teniendo presente los estudios que sobre determinados temas se emprendieron con posterioridad a 1968, como por ejemplo, los realizados sobre los hábitos de consumo de los hogares, el medio ambiente, el turismo, la educación, la salud, el sector público, etc., se consideró necesario aprovechar el concepto de las cuentas satélites para complementar el análisis de las cuentas centrales del PIB.

Las cuentas o sistemas satélites subrayan generalmente la necesidad de ampliar la capacidad analítica de la contabilidad nacional a determinadas áreas de interés social de una manera flexible y sin distorsionar el sistema contable central de las cuentas nacionales.

Por lo general, las cuentas satélites permiten:

1. Proporcionar información adicional de carácter funcional o de entrecruzamiento sectorial sobre determinados aspectos sociales.

2. Emplear conceptos complementarios o alternativos, incluida la utilización de clasificaciones y marcos contables complementarios o alternativos, cuando se necesita introducir dimensiones adicionales en el marco conceptual de las cuentas nacionales.

3. Ampliar la cobertura de los costos y beneficios de las actividades humanas.

4. Incrementar el análisis de los datos mediante indicadores y agregados necesarios.

5. Relacionar las fuentes y el análisis de datos físicos con el sistema contable monetario.

Estas características ponen de manifiesto funciones importantes gracias al análisis de las cuentas satélites. Por una parte, las cuentas satélites están relacionadas con el marco central de las Cuentas Nacionales y, a través de ellas, con el cuerpo principal de las estadísticas económicas integradas. Por otro lado, al referirse específicamente a un campo, también están relacionadas con el sistema de información específico de ese tema. Asimismo, exigen una mejor integración de los datos monetarios y físicos y, dada su estrecha conexión con las Cuentas Nacionales, facilitan el estudio de campos específicos en el contexto de las cuentas y el análisis macroeconómico. Las cuentas satélites pueden ayudar en varios campos a concertar entre sí los análisis de algunos de ellos; por consiguiente, pueden representar un doble papel como herramientas de investigación y como instrumentos de coordinación estadística. 
Algunos de los análisis complementarios o alternativos pueden modificar directa o indirectamente los principales agregados, como se muestra en la contabilidad central de las Cuentas Nacionales. Un ejemplo de modificación directa es cuando se imputan a las Cuentas Nacionales el valor de trabajo en los hogares de las amas de casa. Otros agregados experimentan modificaciones indirectas, como el ingreso disponible como consecuencia de imputarse el valor del trabajo de las amas de casa (lo que se denomina renta ficta).

En general, pueden distinguirse dos tipos de análisis de las cuentas satélites en relación con las cuentas nacionales centrales y la introducción de elementos complementarios que difieren del marco central conceptual (por ejemplo, la identificación de la producción de actividades auxiliares, como el aporte de la industria de restauración al sector turismo), sin que diverjan sustancialmente de los conceptos sobre los que este se constituye. Estas configuraciones no están basadas ni destacan conceptos alternativos, aun cuando los utilizan de manera complementaria.

El primer tipo abarca, en su mayor parte, las cuentas específicas de determinados campos, como los gastos en enseñanza, turismo y protección del medio ambiente. La introducción de su contenido en el marco central de las cuentas podría sobrestimarlo y no sería totalmente confiable; al hacerlo en un marco contable específico, en las cuentas satélites, se incorporan márgenes adicionales de flexibilidad.

El segundo tipo se basa sustancialmente en conceptos alternativos a los aplicados en el Sistema de Cuentas Nacionales. Puede introducirse una frontera de la producción diferente o conceptos ampliados de consumo y formación de capital, o ampliar el ámbito de los activos, o alterar la frontera entre fenómenos económicos y naturales, o situar los vínculos entre el ingreso y la riqueza en el contexto de un concepto más amplio de esta última que incluya los activos naturales, o la riqueza patrimonial producto de la heredad de nuestros antepasados. La utilización de estos conceptos alternativos puede dar lugar a agregados parciales complementarios cuya finalidad es complementar el sistema central.

Este segundo tipo es evidentemente más controvertido que el primero, pero es importante debido a que permite ampliar los trabajos de Cuentas Nacionales, más allá de lo que se incluye o puede incluirse en el sistema nacional de cuentas; ofrece resultados útiles para el análisis económico pues experimenta con nuevos conceptos y metodologías, naturalmente con un margen de libertad mucho mayor que el de los trabajos habituales de Cuentas Nacionales.

A modo de conclusión, podemos afirmar que hoy disponemos de un sistema flexible de Cuentas Nacionales que acepta integrar en ellas las cuentas satélites, permitiéndonos modificar conceptos y agregados finales que difieren del marco central pero que son útiles para los análisis particulares. 


\section{Nota metodológica}

Para efectos de la medición del PIB de cada una de las unidades de producción correspondiente, materia del presente estudio, se utilizó el método de la retribución a los factores de producción. Evitando de esta forma distorsiones en la contabilidad de las cuentas agregadas.

La ventaja que nos otorga este método es el de conocer a ciencia cierta los montos de retribución del PIB generados por cada unidad de producción y su distribución entre los factores mano de obra, capital, retenciones y aportes tributarios al Estado ${ }^{2}$.

\section{Cuentas Satélites de los museos, restos arqueológicos y sitios naturales de las regiones de Arequipa, Cusco, Lambayeque y La Libertad}

\section{Definiciones:}

- El PIB turístico es la unidad de medida monetaria de todos los bienes y servicios finales producidos por el sector turismo, en un período determinado. En resumen, es la sumatoria de los valores monetarios, del consumo de bienes y servicios finales de extranjeros y nacionales, inversión bruta privada, compra de bienes y servicios por parte del Estado, la inversión bruta estatal ${ }^{3}$.

Matemáticamente, podemos definir el Producto Interno Bruto Turístico, con la siguiente ecuación:

$$
\mathbf{P I B}=\mathbf{C}+\mathbf{I}+\mathbf{G}+\mathbf{X}-\mathbf{M}
$$

En donde:

$\mathbf{C}=$ Consumo de nacionales

$\mathbf{I}=\quad$ Inversión Privada Bruta

$\mathbf{G}=$ Gasto público (comprende el gasto en bienes y servicios por parte del Estado, así como la inversión pública estatal)

$\mathbf{X}=$ Exportaciones (consumo turístico de bienes y servicios del receptivo)

$\mathbf{M}=$ Importaciones (insumos, consumo de bienes importados)

Barros de Castro, A. \& Lessa, C. (1995). Introducción a la Economía. México: Siglo Veintiuno Editores S. A. p. 30

Op. cit. p. 127 
- Medición PIB turístico. El PIB turístico se puede medir mediante dos metodologías cuyos resultados matemáticos son exactamente iguales:

1. La primera de ellas es a través de los flujos de productos y servicios finales a precios de mercado ofertados por el turismo.

2. La segunda, mediante la sumatoria de la retribución al costo de los factores de producción de los agentes económicos que intervienen en el sector turismo.

- El valor bruto de la producción turística. Indicamos que el PIB turístico no es otra cosa que la producción de bienes y servicios finales en un período determinado, por lo tanto, el PIB no comprende los bienes intermedios, es decir, aquellos que se utilizan para producir el bien o servicio final.

Sin embargo, en el concepto del valor bruto de la producción, se incluye todo aquello que es vendido por las unidades de producción en forma indiscriminada, sean estos productos y servicios terminados para el consumo o productos que serán utilizados para la producción de nuevos productos o servicios finales.

En resumen, el PIB turístico es el resultado de la diferencia entre el valor bruto de la producción turística y el total de bienes y servicios intermedios producidos para el turismo ${ }^{4}$.

Para efectos de la medición del PIB de cada una de las unidades de producción correspondiente a las unidades de atractivos naturales, museos y restos arqueológicos, se utilizó el método de la retribución a los factores de producción. Evitando de esta forma distorsiones en la contabilidad de las Cuentas Nacionales.

La ventaja que nos otorga este método, es el de conocer a ciencia cierta, los montos de retribución del PIB generados por cada unidad de producción y su distribución entre los factores mano de obra, capital, retenciones y aportes tributarios al Estado ${ }^{5}$. 


\section{Indicadores de los museos, restos arqueológicos y sitios naturales de las regiones de Arequipa, Cusco, Lambayeque y La Libertad}

Las investigaciones realizadas acerca de la generación de la riqueza en los museos, restos arqueológicos y sitios naturales, ubicados en las regiones de Arequipa, Cusco, Lambayeque y La Libertad arrojaron los indicadores que se señalan en la Tabla 1.

Es interesante acotar que los rangos de retribución al factor capital y mano de obra, fluctúan para cada uno de los recursos sujetos a evaluación de conformidad con cada una de las regiones.

Resultan particularmente interesantes los indicadores que en estas investigaciones se han mostrado independientemente de la generación del PIB, su distribución a los distintos factores de producción, el aporte al PIB de cada una de las regiones objeto de las investigaciones. Entre otros indicadores construidos, podemos señalar la cantidad de puestos formales de trabajo ofertados, la demanda o más propiamente dicho la cantidad de visitas. No deja de ser menos interesante las compras intermedias, requeridas por este tipo de unidades de producción turística, las mismas que sumadas al PIB de cada una de estas, nos dan el valor bruto de la producción. Destacamos la importancia de las compras intermedia, ya que de conformidad con lo señalado en la Tabla 1, estas vienen a constituir dentro de una matriz de insumo producto, que es la que se ha elaborado para la obtención de las cuentas satélites del presente estudio, los montos que constituirían el efecto multiplicador a lo largo de la cadena de producción tanto del consumo como de la inversión. 
MARCIA ADRIANA IBERICO DÍAZ, ALEJANDRO MÁLAGA NUÑEZ-ZEBALLOS, JOSÉ MANUEL ENRIQUE MARSANO DELGADO, MARÍA OLIVIA PACHAS FUENTES Y JOSÉ PAZOS MIRANDA

Tabla 1

Indicadores de cuentas satélites de sitios naturales, museos y restos arqueológicos

\begin{tabular}{|c|c|c|c|c|}
\hline Principales indicadores & $\begin{array}{l}\text { Región } \\
\text { Arequipa }\end{array}$ & $\begin{array}{l}\text { Región } \\
\text { Cusco }\end{array}$ & $\begin{array}{c}\text { Región } \\
\text { Lambayeque }\end{array}$ & $\begin{array}{c}\text { Región } \\
\text { La Libertad }\end{array}$ \\
\hline \multicolumn{5}{|l|}{ Atractivos naturales } \\
\hline Porcentaje de atractivos naturales & $100 \%$ & $100 \%$ & $100 \%$ & no aplica \\
\hline Número de trabajadores & 52 & 62 & comunidad & no aplica \\
\hline Número de visitas al año & 395 & 11,021 & 6,036 & no aplica \\
\hline PIB en nuevos soles corrientes & $3,598,723$ & $1,368,541$ & 60,330 & no aplica \\
\hline Compra de bienes intermedios & $11,120,675$ & $-317,895$ & 30 & no aplica \\
\hline Valor Bruto de la Producción & $14,719,398$ & $1,050,646$ & 60,360 & no aplica \\
\hline \multicolumn{5}{|l|}{ Retribución a los factores de producción: } \\
\hline Capital & $59 \%$ & $80 \%$ & $40 \%$ & no aplica \\
\hline Remuneraciones & $41 \%$ & $20 \%$ & $60 \%$ & no aplica \\
\hline Impuestos & no afecto & no afecto & no afecto & no aplica \\
\hline Aporte porcentual al PIB de la región & $0.01 \%$ & $0.01 \%$ & $0.0005 \%$ & no aplica \\
\hline \multicolumn{5}{|l|}{ Museos } \\
\hline Porcentaje de atractivos naturales & $100 \%$ & $100 \%$ & $100 \%$ & $100 \%$ \\
\hline Número de trabajadores & 50 & 47 & 78 & 19 \\
\hline Número de visitas al año & 310,007 & $2,934,316$ & 362,968 & 130,138 \\
\hline PIB en nuevos soles corrientes & $2,353,378$ & $155,292,001$ & $2,205,251$ & 354,850 \\
\hline Compra de bienes intermedios & $4,524,761$ & $39,191,179$ & $1,270,261$ & 901,270 \\
\hline Valor Bruto de la Producción & $6,878,139$ & $194,483,180$ & $3,475,512$ & $1,256,120$ \\
\hline \multicolumn{5}{|l|}{ Retribución a los factores de producción: } \\
\hline Capital & $43 \%$ & $63 \%$ & $13 \%$ & $0 \%$ \\
\hline Remuneraciones & $57 \%$ & $37 \%$ & $87 \%$ & $100 \%$ \\
\hline Impuestos & no afecto & no afecto & no afecto & no afecto \\
\hline Aporte porcentual al PIB de la región & $0.01 \%$ & $1.09 \%$ & $0.02 \%$ & $0.002 \%$ \\
\hline \multicolumn{5}{|l|}{ Restos arqueológicos } \\
\hline Porcentaje de atractivos naturales & $100 \%$ & $100 \%$ & no aplica & $100 \%$ \\
\hline Número de trabajadores & 3 & 398 & no aplica & 54 \\
\hline Número de visitas al año & 23,160 & $8,978,968$ & no aplica & 361,529 \\
\hline PIB en nuevos soles corrientes & 29,580 & $521,594,245$ & no aplica & $1,552,207$ \\
\hline Compra de bienes intermedios & 55,080 & $109,660,647$ & no aplica & $1,454,131$ \\
\hline Valor Bruto de la Producción & 84,660 & $631,254,892$ & no aplica & $3,006,338$ \\
\hline \multicolumn{5}{|l|}{ Retribución a los factores de producción: } \\
\hline Capital & $39 \%$ & $72 \%$ & no aplica & $1 \%$ \\
\hline Remuneraciones & $61 \%$ & $28 \%$ & no aplica & $99 \%$ \\
\hline Impuestos & no afecto & no afecto & no aplica & no afecto \\
\hline Aporte porcentual al PIB de la región & $0.0001 \%$ & $3.66 \%$ & no aplica & $0.01 \%$ \\
\hline
\end{tabular}

Fuente: Antenas del Observatorio Turístico del Perú

Elaboración: Observatorio Turístico del Perú 


\section{Conclusiones}

- Estos estudios, permiten analizar las Cuentas Nacionales, en este caso las regionales, a efectos de escudriñar las mismas en la búsqueda de información cuantitativa sumamente fina sin que se vea alterado el marco central de las Cuentas Nacionales y, en el caso específico las Cuentas Regionales. En tal sentido, conducen al cálculo cierto del PIB generado por el sector hotelero y el de restauración.

- Al sector estatal, lo ayudan en la utilización de una planificación de naturaleza indicativa, para poder diseñar políticas de desarrollo acorde con las políticas de Estado para el sector.

- Al sector privado, le permite el desarrollo de políticas estratégicas de expansión en la inversión.

- Al sector educación, a través de estas investigaciones, le permite brindar a los futuros profesionales una formación mucho más sólida, dándoles una visión de emprendedores internos y externos ya sea que realicen su ejercicio profesional en forma dependiente o independiente.

- Este tipo de estudios permite ir estructurando las distintas matrices de insumo-producto para elaborar las cuentas satélites a nivel de cada una de las unidades de producción que intervienen en la cadena productiva de bienes y servicios del sector turismo. 


\section{Referencias}

Barros de Castro, A. \& Lessa, C. (1995). Introducción a la Economía. Un enfoque estructuralista. México: Siglo Veintiuno Editores. Marsano, J. (2003). Economía del Turismo. Lima: Universidad de San Martín de Porres.

Monitor (1995). Turismo en el Perú. Lima: Comisión de Promoción del Perú.

PromPerú (2011 1a). Perfil del turista extranjero 2010. Lima: Comisión de Promoción del Perú.

PromPerú (2011 1b). Perfil del turista nacional 2010. Lima: Comisión de promoción del Perú.

Scheel, A. (1980). Organización y gestión contable para hoteles y restaurantes. Bogotá: Universidad Externado.

Paginas Web consultadas:

www.bcrp.gob.pe

www.inei.gob.pe

www.observatorioturisticodelperu.com

http://sites.google.com/site/otraucn/investigacion/cstr 